\title{
Relação entre parasitemia de Sauroplasma sp. (Piroplasmorida: Haemohormidiidae) e índices leucocitários em Podocnemis expansa (Tartaruga da Amazônia)
}

A Tartaruga-da-Amazônia (Podocmenis expansa) é um réptil da ordem Chelonia que é encontrada nas bacias do rio Araguaia, Tocantins, Amazonas e Branco. Ela pode medir de $85 \mathrm{~cm}$ a $1 \mathrm{~m}$ e pode pesar até 70 quilos. São a animais com metabolismo lento e que possuem grande expectativa de vida. $O$ gênero Sauroplasma faz parte da ordem Piroplasmida e é um parasita exclusivo de animais de sangue frio parasitando os eritrócitos, mas sua patogenicidade é desconhecida. Neste estudo percebeu-se que a prevalência de Sauroplasma reduziu em relação aos estudos anteriores, e houve um aumento expressivo no número dos leucócitos. Estudos que integram a imunologia e parasitologia são importantes para monitorar e entender os processos patológicos envolvidos na parasitemia de determinados protozoários e como o organismo age na presença de determinados parasitos.

\section{Relationship between parasitemia of Sauroplasma sp. (Piroplasmosoride: Haemohormidiidae) and leukocyte indices in Podocnemis expansa (Amazonian tortoise)}

\begin{abstract}
The Amazon Turtle (Podocmenis expansa) is a reptile of the order Chelonia that is found in the basins of the river Araguaia, Tocantins, Amazonas and Branco. It can measure from $85 \mathrm{~cm}$ to $1 \mathrm{~m}$ and can weigh up to 70 pounds. They are animals with slow metabolism and have great life expectancy. The genus sauroplasm is part of the Pyroplasmide order and is an exclusive parasite of cold-blooded animals parasiting the erythrocytes, but its pathogenicity is unknown.In This study it was perceived that the prevalence ofSauroplasmdecreased in relation to previous studies, and there was a significant increase in the number of leukocytes.Studies that integrate immunology and parasitology are important to monitor and understand the pathological processes involved in the parasitemia of certain protozoa and how the organism acts in the presence of certain parasites.
\end{abstract}

Keywords: Sauroplasm; Turtles; Lymphocytes; Podocnemis expansa; reptiles.

Topic: Conservação da Biodiversidade

Reviewed anonymously in the process of blind peer.
Received: $12 / 04 / 2019$

Approved: 27/05/2019
Aluísio Vasconcelos Carvalho it

Faculdade Guaraí, Brasil

http://lattes.cnpq.br/5200758055263996

http://orcid.org/0000-0002-3793-3133

aluisiovasconcelos@gmail.com

Ana Caroline Lopes da Silva

Faculdade Guaraí, Brasil

http://lattes.cnpq.br/1627109795636932

caroline.energisa@gmail.com

Vanessa Rodrigues Pacheco (id

Faculdade Guaraí, Brasil

http://lattes.cnpq.br/4322373855959265

http://orcid.org/0000-0002-7920-1133

vanessa.rodrigues.pacheco@gmail.com
Referencing this:

CARVALHO, A. V.; SILVA, A. C. L.; PACHECO, V. R.. Relação entre parasitemia de Sauroplasma sp. (Piroplasmorida: Haemohormidiidae) e índices leucocitários em Podocnemis expansa (Tartaruga da Amazônia). Revista Ibero Americana de Ciências Ambientais, v.10, n.3, p.63-73, 2019. DOI: http://doi.org/10.6008/CBPC2179$\underline{6858.2019 .003 .0007}$ 
Relação entre parasitemia de Sauroplasma sp. (Piroplasmorida: Haemohormidiidae) e índices leucocitários em Podocnemis expansa (Tartaruga

\section{INTRODUÇÃO}

Os Testudines são animais da ordem Chelonia, que inclui as tartarugas, jabutis e cágados, ovíparos, seus filhotes se desenvolvem em ovos que são depositados na areia e incubados pela temperatura do ambiente. Esses animais possuem um metabolismo lento e tem uma longa expectativa de vida (OLIVEIRA, 2003; NUNES JUNIOR, 2017).

Cerca de $20 \%$ de todas as espécies mundiais vivem na América do Sul, dentre elas a Podocnemis expansa conhecida como Tartaruga-da-Amazônia (SOUZA, 2004; NUNES JUNIOR, 2017). No Brasil é encontrada nas bacias do rio Araguaia-Tocantins e Amazonas. Pode medir de $85 \mathrm{~cm}$ a $1 \mathrm{~m}$ de comprimento e pesar até $70 \mathrm{~kg}$, tornando-se o maior quelônio de água doce da América do Sul, possui coloração verde-oliva acinzentada e atinge maturação sexual entre 7 e 8 anos de idade (IBAMA, 2007; OLIVEIRA, 2003; FREITAS et al., 2007; NUNES JUNIOR, 2017).

As primeiras pesquisas com células sanguíneas em répteis data no início do século XVII, com o surgimento do microscópio, realizando os primeiros estudos hematológicos em serpentes (GULLIVER, 1842). No ano de 1879, Havem estudou o sangue de algumas espécies de répteis e anfíbios e definiu o número e proporções de eritrócitos e leucócitos. Em 1930, surgiu o primeiro trabalho elaborado comparando a hematologia das espécies de lagartos, tartarugas e serpentes (BABUDIERI, 1930).

O parasitismo é uma ligação estreita e direta no convívio de dois organismos devidamente determinados: o hospedeiro e o parasita, vivendo o segundo à custa do primeiro. Espécies parasitas necessitam manter seus hospedeiros vivos, em razão de necessitar de boas condições para sua subsistência, visto que esses últimos proporcionam abrigo, alimento e condições para a reprodução dos parasitos (PESSÔA et al., 1982; POUGH et al., 2004; LEGLER et al., 2013). A quantidade de parasitas em um hospedeiro é controlada pelos fatores genéticos e a resposta do hospedeiro em relação aos parasitas. Alguns animais podem ser mais predispostos a infeções devido a fatores genéticos, nutricionais ou ambientais (TIZARD, 2014).

Os parasitos do gênero Sauroplasma (TOIT, 1937) fazem parte da ordem Piroplasmida, e são pertencentes à família Haemohormidiidae, assim como os gêneros Serpentoplasma e Haemohormidium (LEVINE, 1980; READEL et al., 2008). Segundo Toit (1937), o protozoário pode possuir forma irregular ou arredondada, onde a forma prevalente é a de um anel com um grande vacúolo central associado a grânulos de cromatina. A reprodução ocorre por fissão binária ou brotamento. Piroplasmas podem aparecer em eritrócitos como pequenas inclusões medindo entre 1,1-5,0 × 1.0- $4.3 \mu \mathrm{m}$ (LAINSON et al., 1971; BENNET et al., 1976).

A ocorrência de Sauroplasma em répteis é bastante comum e com ampla distribuição geográfica, onde formas parasitárias têm sido registradas em diferentes famílias de lagartos na Europa, África, Ásia e nas Américas (TELFORD, 2016; UILENBERG et al., 1966; FRANK, 1974; ALBERTS et al., 1998). Entretanto, seu ciclo de vida, patogenicidade e vetores são desconhecidos (DAVIES et al., 2000; TELFORD, 2016). É provável que os vetores para répteis terrestres sejam carrapatos e ácaros devido à presença destes em lagartos infectados, 
mas para répteis aquáticos o vetor é totalmente desconhecido (PIENAAR, 1962; UILENBERG et al., 1966; ALBERTS et al., 1998; READEL et al., 2008; TELFORD, 1984; 2016).

Estudos experimentais evidenciam que Sauroplasma pode ser transmitido por sanguessugas Unoculubranchiobdella expansa, espécie encontrada em grandes quantidades parasitando Tartarugas da Amazônia e Tracajás e com alta prevalência do protozoário nas populações naturais (CARVALHO et al., 2018). O objetivo desse trabalho é avaliar a parasitemia por Sauroplasma e relacionar o número e caracterização de leucócitos por animal.

\section{METODOLOGIA}

No decorrer da pesquisa foram amostrados 06 exemplares de $P$. expansa. Os filhotes foram acomodados em caixas plásticas e transportados para o Laboratório de Análises Clínicas do IESC/Faculdade Guaraí para coleta de sangue. A largura e comprimento da carapaça, o peso, dentre outros aspectos foram medidos de acordo com a metodologia de Malvasio et al. (2002).

Os répteis foram submetidos a uma colheita de sangue para amostras a partir da veia da cauda ou ventral ou o seio subcarapacial e colocadas em tubos revestidos com heparina de lítio para esfregaços sanguíneos, logo após, foram fixados com metanol e corados com Giemsa a 10\% (EISEN et al., 2000; HERNANDEZ-DIVERS et al., 2002; FALCE, 2009).

As informações morfométricas das formas parasitárias achadas foram registradas e expressas em $\mu \mathrm{m}$. O nível de parasitemia foi estimada pela contagem das formas parasitárias em 20 réplicas de 100 eritrócitos (RBC) por campo, totalizando aproximadamente 2.000 eritrócitos (GODFREY et al., 1987; 1990). Esta pesquisa contou com as autorizações do Instituto Brasileiro do Meio Ambiente e dos Recursos Naturais Renováveis - IBAMA (SISBIO № 46928-1/ 2014) e do Comitê de Ética em Experimentação Animal da Universidade Federal de Tocantins (№ 23101.002459/2015-99).

\section{RESULTADOS E DISCUSSÃO}

\section{Biometria}

Os animais amostrados foram submetidos a biometria avaliando o comprimento da carapaça (CCAR), largura da carapaça (LCAR), comprimento do plastrão (CPLA), largura do plastrão (LPLA), abertura plastrãocarapaça (APC) e massa corporal (em kg) (tabela 1). Observou-se que os filhotes não apresentaram mudança significativa na biometria entre os indivíduos amostrados apresentando média de 15,6 $\pm 0,9$ em LCAR, 17,7 \pm 1,3 em CCAR, 13,9 \pm 0,9 em CPLA, 10,3 \pm 0,6 em LPLA e 3,5 \pm 0,3 em APC. Não houve relação entre o peso (massa corporal) e parasitemia. Os animais são alimentados regularmente com ração de peixe a $30 \%$ de proteína, se igualando ao teor proteico dos animais em ambientes naturais. Dessa forma, os indivíduos não apresentam tamanhos variados significativos já que recebem alimento no mesmo período e na mesma quantidade. 
Tabela 1: Dados morfométricos dos indivíduos amostrados em $P$. expansa.

\begin{tabular}{|l|l|l|l|l|l|l|}
\hline Animal & LCAR & CCAR & CPLA & LPLA & APC & Massa (g) \\
\hline 01 & $15,5 \mathrm{~cm}$ & $17,3 \mathrm{~cm}$ & $13,8 \mathrm{~cm}$ & $10 \mathrm{~cm}$ & $3,4 \mathrm{~cm}$ & 570 \\
\hline 02 & $15,2 \mathrm{~cm}$ & $17,6 \mathrm{~cm}$ & $13,6 \mathrm{~cm}$ & $10,2 \mathrm{~cm}$ & $4 \mathrm{~cm}$ & 570 \\
\hline 03 & $16,3 \mathrm{~cm}$ & $18,5 \mathrm{~cm}$ & $14,4 \mathrm{~cm}$ & $10,6 \mathrm{~cm}$ & $3,4 \mathrm{~cm}$ & 640 \\
\hline 04 & $16,6 \mathrm{~cm}$ & $19 \mathrm{~cm}$ & $14,3 \mathrm{~cm}$ & $10,5 \mathrm{~cm}$ & $3,5 \mathrm{~cm}$ & 550 \\
\hline 05 & $16 \mathrm{~cm}$ & $18,5 \mathrm{~cm}$ & $14,7 \mathrm{~cm}$ & $11 \mathrm{~cm}$ & $3,5 \mathrm{~cm}$ & 640 \\
\hline 06 & $14 \mathrm{~cm}$ & $15,5 \mathrm{~cm}$ & $12,3 \mathrm{~cm}$ & $9,2 \mathrm{~cm}$ & $3,2 \mathrm{~cm}$ & 410 \\
\hline
\end{tabular}

\section{Prevalência}

Durante a pesquisa foi estudado seis animais da espécie Podocnemis expansa e todos os indivíduos se encontram parasitados por Sauroplasma (figura 1).

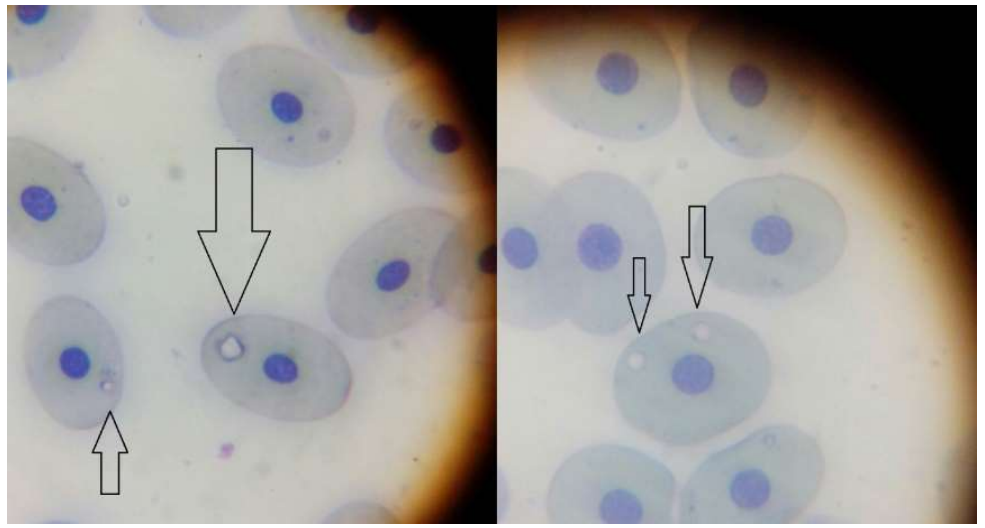

Figura 1: Sauroplasma parasitando hemácia de p. expansa.

Observou-se que o número de parasitos declinou (figura 2) evidenciando que tal protozoário pode ser combatido pelas células de defesa, garantindo a eficiência das barreiras naturais.

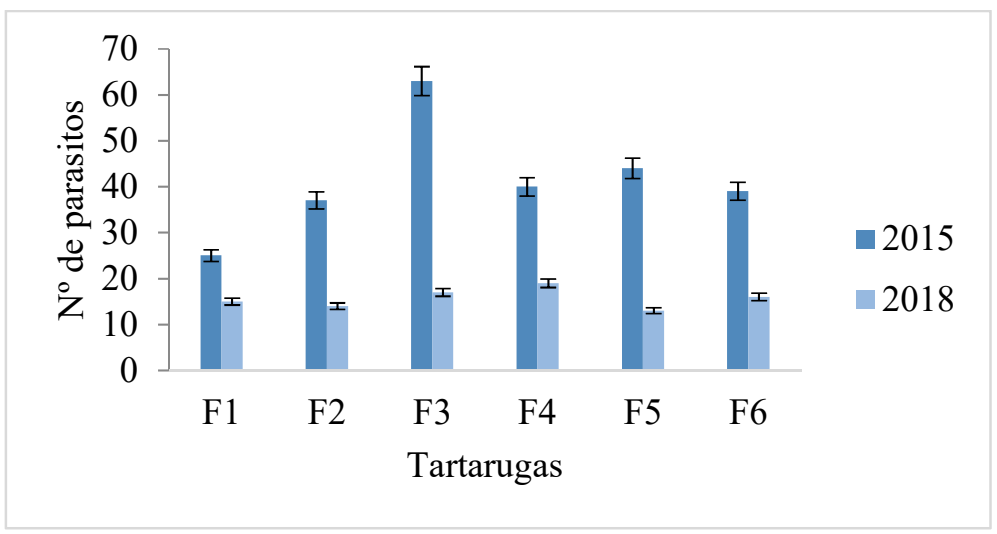

Figura 2: Prevalência de Sauroplasma em Podocnemis expansa.

Existem diversas condições que determinam diferentes desfechos em uma infeção (cura, doença aguda e morte, infecção crônica, doença subclínica ou assintomática) e possuem influências multifatoriais complexas interligadas diretamente ao hospedeiro (situação imunológica do indivíduo e da população, período de exposição ao parasito, estresse, sexo, fatores genéticos, densidade populacional), ao parasito (cepa, virulência), ao ambiente em que estes organismos estão inseridos (STALLONES, 1972; YORKE et al., 1979; HIRSH et al., 2003).

Uma das mais frequentes causas de doenças ou processos patológicos entre as tartarugas estão às 
Relação entre parasitemia de Sauroplasma sp. (Piroplasmorida: Haemohormidiidae) e índices leucocitários em Podocnemis expansa (Tartaruga

péssimas condições de manejo, a que na maioria das vezes são submetidas. As enteroparasitoses e hemoparasitoses podem ser responsáveis por certo grau de anemia e até mesmo a morte. São inúmeras as espécies de parasitas que acometem o sangue e o tubo digestivo dos quelônios, entre os quais destacam-se protozoários, hirudíneos, helmintos e acantocéfalos, sendo que alguns destes podem determinar patologias graves nos animais (BOURDEAU, 1988).

Outras espécies de quelônios possuem vulnerabilidade diferente em relação a parasitose, dentre elas estão as condições ambientais como a temperatura e a pluviometria, a exposição aos parasitos, fatores comportamentais como a junção de uma colônia, e genético tal como a imunidade (LEE et al., 2006; MARINI, 2001; BEGON et al., 2007; CARVALHO, 2016).

Nos estudos evidenciados em outros répteis não há parasitose por Sauroplasma nos indivíduos amostrados, o que mostra que o protozoário não seja patogênico. Segundo Telford (2009) o Sauroplasma, mesmo sendo um piroplasmida, possui pouca afinidade com outros representantes do mesmo grupo como Babesia e Theileria, protozoários encontrados em mamíferos e patogênicos, o que reduz o parasitismo em alguns grupos animais. Da mesma maneira, estudos relatados por Van (2012) evidencia que as infecções causadas por Sauroplasma parecem ter pouco efeito visível no próprio hospedeiro.

\section{Parasitemia}

No período de 2018 a média parasitária foi de 15,7 $\pm 2,2$ dos animais amostrados (CARVALHO et al., 2018). Os mesmos animais foram submetidos anteriormente à pesquisa constatando a parasitemia média de $41,3 \pm 12,4$ nos filhotes de $P$. expansa. A parasitemia média dos filhotes foi de $41 / 2.000$ (2\%) dos eritrócitos em 2015, no estudo realizado em 2018 o valor foi de 15/2.000 (0,7\%). No estudo realizado por Picelli et al. (2016) a taxa média de parasitemia foi de 44,14/2.000 eritrócitos (2,2\%) nas populações amostradas de Podocnemis expansa., evidenciando que o protozoário não gera parasitemias altas em seus hospedeiros.

Sabe-se que em animais de vida livre as informações obtidas são poucas e inadequadas para um bom diagnóstico, por isso é feito o exame sanguíneo (SANTOS et al., 2005). A hematologia é a técnica mais utilizada na medicina silvestre e o uso do esfregaço sanguíneo é o melhor método de diagnosticar previamente qualquer alteração no corpo do animal (CAMPBELL, 2006; CUNHA, 2013).

Normalmente a interpretação dos testes bioquímicos em sangue de répteis é semelhante aos descritos para mamíferos domesticados. No entanto é considerada as alterações externas (como as ambientais, condições nutricionais, estação do ano) que podem ter maior ou menor influência na saúde e fisiologia de animais ectotérmicos (THRALL, 2007).

No presente estudo as análises sanguíneas foram feitas através da microscopia óptica, considerada a metodologia padrão para o diagnóstico de hemoparasitos (SEGHAL et al., 2005), onde constatou-se que houve diminuição dos parasitas em relação ao hospedeiro, isto mostra que os animais estão desenvolvendo imunidade contra os parasitas.

Como se sabe, os leucócitos possuem a função de identificar e de combater os invasores externos ao 
corpo e os heterófilos estão associados a infecção parasitária (FUDGE, 2000; KERR, 2003; LORENZI, 2003; OZZETTI, 2013). Devido à falta de variabilidade genética dos parasitos em detrimento da manutenção parasitária, faz com que o sistema imune consiga eliminar com mais eficiência os protozoários encontrados no hospedeiro, a ausência de vetores que completam o ciclo do parasita compromete a população natural de Sauroplasma no organismo.

Os parasitos possuem diversas estratégias de exploração de hospedeiros, podendo variar do completo generalismo ao alto grau de especialização, porém tais comportamentos são dependentes de complexas interações entre fatores bióticos e abióticos nas comunidades onde a transmissão ocorre (RICKLEFS et al., 2002). Os fatores abióticos são determinados pelas condições climáticas e as características do hábitat, determinando a diversidade e a abundância dos hospedeiros vertebrados e invertebrados. Os fatores bióticos, relacionados aos hospedeiros, incluem sexo, faixa-etária, imunidade, padrões de migração e de comportamento (BENSCH et al., 2000; RICKLEFS et al., 2005).

Outro fator que pode ter influenciado a diminuição de Sauroplasma é a ausência do vetor na manutenção do ciclo parasitário, pois em répteis terrestres o protozoário é transmitido provavelmente por carrapatos e ácaros, que são encontrados nos animais parasitados. Já em animais aquáticos o vetor é desconhecido, Carvalho et al. (2018) em seu experimento mostrou que é muito provável que a transmissão do parasita seja feita por sanguessugas, já que os filhotes usados no estudo não encontravam se parasitados anteriormente.

Estudos voltados para este tema indicam que a diversidade onde esses animais se encontram reduz o risco de se obter a doença, quando é redistribuído as possíveis fontes de alimentação do vetor, a chance de alcançar o hospedeiro ideal é menor (CARVALHO, 2016). Os animais utilizados neste estudo não apresentam qualquer tipo de contato com vetores de transmissão, o que faz com que a resposta imune seja maior, já que os hospedeiros não estão tendo novas infecções e por isso estão conseguindo criar imunidade e combater o invasor.

\section{Leucócitos}

Decidiu-se fazer análise sanguínea de seis exemplares que já possuíam o protozoário por meio de infecção experimental, os mesmos indivíduos avaliados no estudo de Carvalho (2016) e que não haviam sido expostos a outros hematozoários e ectoparasitos durante a pesquisa para verificar como as células de defesa (índices leucocitários) estavam se comportando. $\mathrm{Na}$ amostra examinada, verificou a presença de leucócitos nos animais amostrados. As células de defesa encontradas foram contadas em um campo de 100 células, obtendo assim os valores mencionados na tabela abaixo (tabela 2). Não foram observadas evidências sugestivas de eosinófilos no material examinado.

De acordo com Stacy et al. (2011) os leucócitos dos répteis podem ser classificados em granulócitos (basófilos, heterófilos e eosinófilos), e podem ainda ser divididos em duas classes: acidófilos e basófilos, classificados de acordo com a aparência em esfregaço sanguíneo. Existe um grade desafio na mensuração de 
valores quantitativos de leucócitos em répteis visto que a literatura apresenta valores discrepantes, possivelmente devido à grande variação entre indivíduos da mesma faixa etária (PIRES et al., 2009).

Tabela 2: Leucócitos totais nos filhotes amostrados (campo de 100 células).

\begin{tabular}{|l|l|l|l|l|l|}
\hline Filhotes & Leucócitos & Monócitos & Trombócitos \\
\hline & Heterófilos & Azurófilos & Linfócitos & Mon & 10 \\
\hline 01 & 43 & 11 & 33 & 3 & 7 \\
\hline 02 & 42 & 11 & 32 & 3 & 6 \\
\hline 03 & 43 & 10 & 38 & 3 & 5 \\
\hline 04 & 47 & 11 & 34 & 3 & 8 \\
\hline 05 & 45 & 8 & 36 & 3 & 10 \\
\hline 06 & 41 & 11 & 30 & 8 & \\
\hline
\end{tabular}

Em todos os animais foram encontrados grande número de heterófilos e linfócitos (figura 3) e em menor quantidade azurófilos, trombócitos, e monócitos respectivamente. $\mathrm{O}$ sangue dos répteis é constituído por eritrócitos nucleados, monócitos, eosinófilos, heterófilos, trombócitos, basófilo e linfócitos (CAMPBELL, 2006).

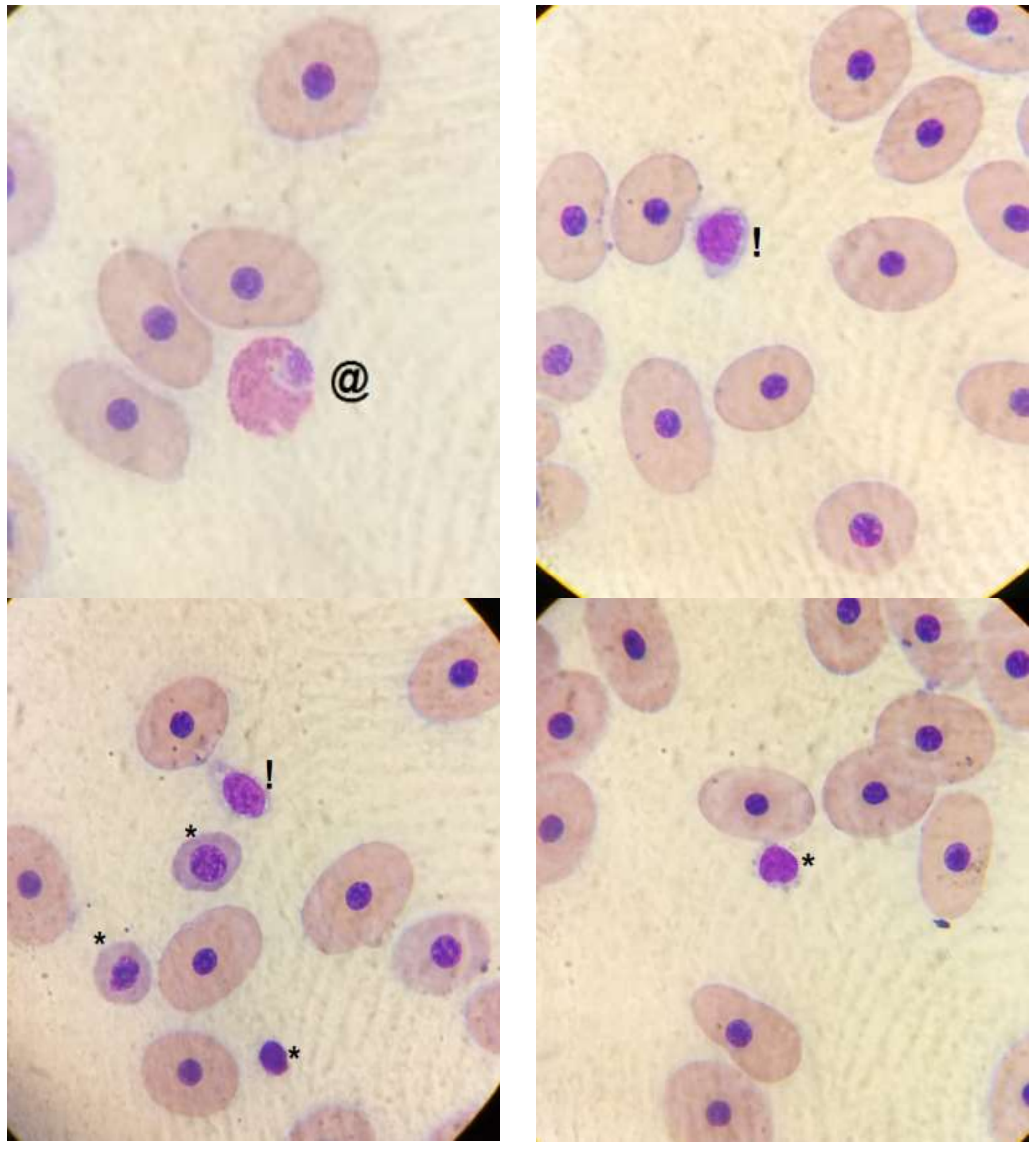

Figura 3: Fotos de hemácias e células de defesa.

Legenda: Linfocitos (*), Monócitos (!) , @ Heterófilo (@).

Algumas doenças podem se manifestar no exame de sangue, sendo assim necessário realizar outros exames laboratoriais. Quando o animal está anêmico pode haver variação nos níveis de eritrócitos, e o aumento ou diminuição dos leucócitos pode ou não indicar infecção ou doença viral (FALCE, 2009). Os linfócitos são células de defesa do organismo com forma arredondada e seu tamanho pode variar entre 05 $\mu \mathrm{m}$ e $15 \mu \mathrm{m}$, possui núcleo esférico que ocupa o centro da célula sendo envolto por citoplasma basófilo homogêneo. São produzidos em grande quantidade quando o hospedeiro está infectado, assim 
Relação entre parasitemia de Sauroplasma sp. (Piroplasmorida: Haemohormidiidae) e índices leucocitários em Podocnemis expansa (Tartaruga

correspondendo como indicador da saúde do animal (PINTO, 2017).

Os linfócitos são células do sistema inume, conhecidas também como células imunocompetentes (JAMRA et al., 1983; OZZETTI, 2013). Existem três subtipos de linfócitos: Linfócito B, Linfócito T e natural killer (NK), os linfócitos B e T são responsáveis pelas respostas imunológicas celulares e humorais, são as células de reconhecimento de agentes externos, os linfócitos T agem diretamente sobre o invasor e os linfócitos B produzem anticorpos (LICHTMAN et al., 2005; HAMSEL et al., 2007; LORENZI, 2003; OZZETTI, 2013).

As células dos répteis costumam ter uma grande variação morfológica, o que prejudica na identificação dos leucócitos. Está identificação também é dificultada pela grande variedade de nomenclatura que atualmente tem na literatura. Normalmente os leucócitos dos répteis são divididos em granulócitos e não granulócitos. Os linfócitos encontrados em sangue de répteis são similares ao que são encontrados em aves e mamíferos, sendo rotulado de igual forma (PINTO, 2017).

Os heterófilos são células que possuem forma esférica, ovalada ou irregular. Possui um citoplasma abundante com grânulos acidófilos compactos e irregulares e contém núcleo circular (OZZETTI, 2013). Quando há aumento dessa célula é sinal de infecção por parasitas, a função dos heterófilos é fagocitar os agentes invasores (FUDGE, 2000)

Como visto, estas células estão intimamente ligadas com as defesas dos organismos, e o seu aumento pode indicar infecção parasitária pelo fato de que quanto mais células de defesa maior é a quantidade de invasores ou maior é a defesa do organismo contra o ser invasor (leucocitose). Diversos estudos na literatura da parasitologia têm atentado para os motivos e consequências do modelo de compartilhamento dos parasitas em vetores (ANDERSON et al., 1982). No entanto, a ligação entre distribuição da quantidade de parasitas por vetor e a sua respectiva idade ainda não foram mensuradas e definidas.

O conjunto dos parasitas na população hospedeira pode crescer com a baixa idade pois o vetor fica vulnerável. Com o amadurecimento do hospedeiro, o conjunto dos parasitas pode regressar pelo o aumento na vulnerabilidade dos vetores e uma baixa na mortalidade dos parasitas, porém o conjunto dos parasitas volta a cair em vetores com faixa etária mais alta (ERNEST et al., 1989; SOUZA, 2004; SOUZA et al., 1988).

A nutrição possivelmente seja um dos aspectos com maior frequência em estudos e biografias dos quelônios do Brasil, e pode ter uma relação intrínseca com a imunidade, isso porque a espécie $P$. expansa muda sua alimentação no período de seca e cheia do rio e durante sua fase de crescimento (ERNEST et al., 1989; SOUZA, 2004; SOUZA et al., 1988). Certamente acontecem alterações ontogênicas na dieta, possivelmente rica em proteína contribuindo no crescimento durante a fase jovem.

A dificuldade maior na dieta das tartarugas é a quantidade de proteínas que elas conseguem em vida livre, as espécies criadas em cativeiro recebem uma dieta baixa em proteínas que são necessárias para a sobrevivência e crescimento. As pesquisas realizadas a respeito da ecologia nutricional além de esclarecer o modo como os organismos das espécies necessitam ainda são carentes principalmente sobre as funções biológicas e compreensão de seus nichos (DRESLIK, 1999). 
Relação entre parasitemia de Sauroplasma sp. (Piroplasmorida: Haemohormidiidae) e índices leucocitários em Podocnemis expansa (Tartaruga da Amazônia)

\section{CONCLUSÕES}

Pode-se concluir que os hospedeiros que não se encontram mais em contato com o possível vetor (sanguessuga) conseguem criar anticorpos suficientes para eliminar o parasita. A alimentação pode ter também influenciado no aumento da imunidade dos animais, pois um animal saudável produz uma melhor reação imunológica.

A parasitemia é influenciada pela manutenção dos vetores no hospedeiro, se o ciclo é quebrado, os parasitas não mantem sua população reduzindo o número de protozoários sobre o hospedeiro. Os leucócitos possuem uma importância primordial no combate aos invasores detectando-os e eliminando à medida em que são encontrados.

\section{REFERÊNCIAS}

ANDERSON, R. M.. Parasite dispersion patterns: generative mechanisms and dynamic consequences. In: MEEROVITCH, E.. Aspects of parasitology. Montreal, McGill University Press, 1982.

ALBERTS, A. C.; OLIVA, M. L.; WORLEY, M. B.; TELFORD, S. R. The need for pre-release health screening in animal translocations: a case study of the Cuban iguana (Cyclura nubila). Animal Conservation, v.1, n.3, p.165-172, 1998.

BABUDIERI, B.. Studi di ematologia comparata. Haematologica, v.11, p.199-255, 1930.

BEGON, M.; TOWNSEND, C. R.; HARPER, J. L.. Ecologia: de indivíduos a ecossistemas. 4 ed. Porto Alegre: Artmed, 2007.

BENNETT, G. F.; BORRERO H. J. I.. Blood parasites of some birds from Colombia. Journal of Wildlife Diseases, v.12, n.3, p.454-458, 1976.

BENSCH, S.; STJERNMAN, M.; HASSELQUIST, D.; OSTMAN, O.; HANSON, B.; WESTERDAHL, H.; PINHEIRO, R. T.. Host specificity in avian blood parasites: a study of Plasmodium and Haemoproteus mitochondrial DNA amplified from birds. Proceedings of the Royal Society of London. Series B: Biological Sciences, v.267, n.1452, p.1583-1589, 2000.

BOURDEAU, P.. Pathologie des tortues, 2e partie: affections cutanées et digestives. Le Point vétérinaire: revue d'enseignement post-universitaie et de formation permanente, v.20, n.118, p.19-32, 1988.

CAMPBELL, T. W.. Clinical pathology of Reptiles. In: MADER, D. R.. Reptile Medicine and Surgery. Florida: Sauders Elsevier, 2006. p.453-470.

CARVALHO, A. V.. Infecção experimental em Podocnemis expansa (Testudines, Podocnemididae) e importância social da espécie no Rio Javaés, Tocantins, Brasil. Dissertação (Mestrado em Ciências do Ambiente) Universidade Federal do Tocantins, 2016.

CARVALHO, A. V.; MALVASIO, A.. Transmissão de Sauroplasma sp. (Piroplasmorida: Haemohormidiidae) pela sanguessuga Unoculubranchiobdella expansa (Hirudinea: Ozobranchidae) em Podocnemis expansa (Tartaruga da
Amazônia). Revista Ibero-Americana de Ciências Ambientais, v.9, n.2, 2018.

CUNHA, D. M.; BRAGA, R. R.; MOTA, J. F.. O esfregaço sanguíneo como diagnóstico preliminar em quadro clínico inespecífico de Mesoclemmys tuberculata Luederwaldt, 1926 (Testudines, Chelidae). In: SIMPÓSIO CEARENSE DE ANIMAIS SELVAGENS, 3. Anais. Grupo de Estudos de Animais Selvagens do Ceará, 2013. p.58-60

DAVIES, A. J.; JOHNSTON, M. R. L.. The biology of some intraerythrocytic parasites of fishes, amphibia and reptiles. Advances in parasitology, v.45, p.1-107, 2000.

DRESLIK, M. J.. Dietary notes on the red-eared slider (Trachemys scripta) and river cooter (Pseudemys concinna) from southern Illinois. Transactions of the Illinois State Academy of Science, v.92, n.3-4, p.233-241, 1999.

EISEN, R. J.; SCHALL, J. J.. Life history of a malaria parasite (Plasmodium mexicanum): independent traits and basis for variation. Proceedings of the Royal Society of London B: Biological Sciences, v.267, n.1445, p.793-799, 2000.

ERNEST, C. H.; BARBOUR, R. W.. Turtles of the World. 1989.

FALCE. M. C. L. B.. Hematologia de Répteis: Revisão Bibliográfica. Monografia (Especialização em Clínica Médica e Cirúrgica de Animais Selvagens e Exóticos) - Universidade Castelo Branco, Campinas, 2009.

FUDGE, A. M.. Laboratory medicine avian and exotic pets. Philadelphia: W. B. Sauders Company, 2000.

FRANK, W.. Sauroplasma and other so-called parasites of reptile blood corpuscles. Proc. 3rd Int. Congr. Parasitol, v.3, p.1666, 1974.

FREITAS, M. A.; SILVA, T. F. S.. Herpetofauna das caatingas e áreas de altitudes do Nordeste brasileiro. Pelotas: USEB, 2007.

GODFREY JR, R. D.; FEDYNICH, A. M.; PENCE, D. B..

Quantification of hematozoa in blood smears. Journal of Wildlife Diseases, v.23, n.4, p.558-565, 1987. 
GODFREY JR, R. D.; PENCE, D. B.; FEDYNICH, A. M.. Effects of host and spatial factors on a haemoproteid community in mourning doves from western Texas. Journal of Wildlife Diseases, v.26, n.4, p.435-441, 1990.

GULLIVER, G.. On the blood corpuscles of the british ophidians, reptiles and other oviparous vertebrates. Proc. Zool. Soc. Lon. v.10, p.107-112, 1842.

HANSEL, D. E.; DINTZIS, R. Z.. Fundamentos de Rubin: Patologia. Rio de Janeiro: Guanabara Koogan, 2007.

HERNANDEZ-DIVERS, S. M.; HERNANDEZ-DIVERS, S. J.; WYNEKEN, J.. Angiographic, anatomic and clinical technique descriptions of a subcarapacial venipuncture site for chelonians. Journal of Herpetological Medicine and Surgery, v.12, n.2, p.32-37, 2002.

HIRSH, D. C.; ZEE, Y. C.. Microbiologia veterinária. Rio de Janeiro: Guanabara Koogan, 2003.

IBAMA. Instituto Brasileiro do Meio Ambiente e dos Recursos Naturais Renováveis. Espécies Manejadas. 2007.

JAMRA, M.; LORENZI, T. F.. Leucócitos, leucemias e linfomas. Rio de Janeiro: Guanabara Koogan, 1983.

KERR, M. G.. Exames Laboratoriais em Medicina Veterinária. 2 ed. São Paulo: Roca, 2003.

LAINSON, R.; LANDAU, I.; SHAW, J. J.. On a new family of non-pigmented parasites in the blood of reptiles: Garniidae fam. nov.,(Coccidiida: Haemosporidiidea). Some species of the new genus Garnia. International Journal for Parasitology, v.1, n.3, p.241-250, 1971.

LEE, K. A.; MARTIN, L. B.; HASSELQUIST, D.; RICKLEFS, R. E.; WIKELSKI, M.. Contrasting adaptive immune defenses and blood parasite prevalence in closely related passer sparrows. Oecologia, p.537-546, 2006.

LEGLER, J. M.; VOGT, R. C.. Natural Predators na Parasites. In: The turtlesos Mexico: Land and fresshwater forms. University of California Press, 2013.

LEVINE, N. D.; CORLISS, J. O.; COX, F. E. G.; DEROUX, G.; GRAIN, J.; HONIGBERG, B. M.; MERINFELD, E. G.. A newly revised classification of the protozoa. The Journal of protozoology, v.27, n.1, p.37-58, 1980.

LORENZI, T. F.. Manual de Hematologia: Propedêutica e Clínica. 3 ed. Medsi: 2003.

MALVASIO, A.; SOUZA, A. M.; FERREIRA JUNIOR, P. D.; REIS, E. S.; SAMPAIO, F. A. A.. Temperatura de incubação dos ovos e granulometria dos sedimentos das covas relacionadas a determinação sexual em Podocnemis expansa (Schweigger, 1812) e $P$. unifilis (Troschel, 1848) (Testudines,

Pelomedusidae). Publicações Avulsas, n.5, p.11-25, 2002.

MARINI, M. Â.; DURÃES, R.. Annual cycles of molt and reproduction of passerines from central-south Brazil. Condor, v.103, n.4, p.767-775, 2001.

NUNES JÚNIOR, F. P.. Hematologia e bioquímica sérica de Testudines continentais brasileiros em cativeiro.
Dissertação (Programa de Pós-Graduação em Recursos Pesqueiros e Aquicultura) - Universidade Federal Rural de Pernambuco, Recife, 2017.

OLIVEIRA, O. M. A.. Animais Silvestres e Exóticos. São Paulo: Roca, 2003

PIRES, T. T.; ROSTAN, G.; BITTENCOURT, T. C. C.; GUIMARÃES, J. E.. Hemograma e Bioquímica Sérica de tartarugas Cabeçudas (Caretta) de vida Livre e mantidas em cativeiro, no Litoral Norte da Bahia. Braz. K. Vet. Res. Sci, v.46, n.1, p.11-18, 2009.

OZZETTI, P. A.. Hematopoese em serpentes Oxyrhopus guibei (Hoge \& Romano, 1978) (Ophidia: Dipsadidae): caracterização morfológica, citoquímica e ultraestrutural. Tese (Doutorado em Ciências) - Universidade de São Paulo, São Paulo, 2013.

PESSÔA, S. B.; MARTINS, A. V.. Introdução à Parasitologia: Parasitologia Médica. 11 ed. Guanabara Koogan, 1982.

PICELLI, A. M.; CARVALHO; A. V.; VIANA, L. A.; MALVASIO, A.. Parasitization by Sauroplasma sp. (Apicomplexa: Haemohormidiidae) in Chelonian Podocnemis expansa (Testudines: Podocnemididae) in the Brazilian Amazon. Journal of Parasitology, v.102, n.1, p.161-165, 2016.

PIENNAR, U. V.. A preliminary note on reptilian haemoprotozoa in South Africa. In: Haematology of some South African reptiles. Witwatersrand Univ. Press, Johannesberg, 1962.

PINTO, F. F.. Hematologia em Aves e Répteis: Atlas colorido. Relatório final de estágio. (Mestrado Integrado em Medicina Veterinária) - Universidade de Porto, Porto, 2017.

POUGH, F. H.; ANDREWS, R. M.; CADLE, J. E.; CRUMP, M. L; SAVITZKY, A. H.; WELLS, K. D.. Herpetology. 3 ed. Prentice Hall: Upper Saddle River, 2004.

READEL, A. M.; PHILLIPS, C. A.; WETZEL, M. J.. Leech parasitism in a turtle assemblage: effects of host and environmental characteristics. Copeia, v.2008, n.1, p.227233,2008 .

RICKLEFS, R. E.; FALLON, S. M.. Diversification and host switching in avian malaria parasites. Proceedings of the Royal Society of London B: Biological Sciences, v.269, n.1494, p.885-892, 2002.

RICKLEFS, R. E.; SWANSON, B.; FALLON, S. M.; MARTÍNEZABRAÍN, A.; SCHEUERLEIN, A.; GRAY, J.; LATTA, S. C.. Community relationships of avian malaria parasites in southern Missouri. Ecological Monographs, v.75, n.4, p.543559, 2005.

SANTOS, A. L. Q.; MALTA, T. S.; MUNDIM, A. V.; JÚNIOR, J. A.; CARVALHO, S. F. M.. Variação dos Constituintes Bioquímicos e Sanguíneos de Tartarugas-da-Amazônia (Podocnemis expansa, Schweigger- 1812) (testudinata) Mantidas em Criatório Comercial. Archives of Veterinary Science, v.10, n.3, p.1-8, 2005. 
SEHGAL, R. N.; JONES, H. I.; SMITH, T. B.. Blood parasites of some West African rainforest birds. Journal of Veterinary Medicine Sciese, v.67, p.295-301, 2005.

SOUZA, F. L.. Uma Revisão sobre Padrões de Atividade, Reprodução e Alimentação de Cágados Brasileiros (Testudines, Chelidae). Phyllomedusa, v.3, n.1, p.15-27, 2004.

SOUZA, V. L.; URBINATI, E. C.; MARTINS, M. I. E. G.; SILVA, P. C.. Avaliação do crescimento e do custo da alimentação do pacu (Piaractus mesopotamicus Holmberg, 1887) Submetido a ciclos alternados de restrição alimentar e realimentação. Revista Brasileira de Zootecnia, v.32, n.1, p.1-15, 1998.

STACY, N. I.; ALLEMAN, A. R.; SAYLER, K. A.. Diagnostic Hematology of Reptiles. Clinics in Laboratory Medicine, v.31, p.87-108, 2011.

STALLONES, R. A.. Environment, ecology, and epidemiology. Who Chronicle, v.26, n.7, p.294, 1972.

TELFORD JUNIOR, S. R.. Haemoparasites of reptiles In: HOFF, G. L.; FRYE, F. L.; JACOBSON, E. R.. Diseases of amphibians and reptiles. New York: Plenum Press, 1984.

TELFORD JUNIOR, S. R.. Hemoparasites of the Reptilia: Color Atlas and Text. Boca Raton: CRC Press, 2009.
TELFORD JR, S. R.. Hemoparasites of the Reptilia: Color atlas and text. Boca Raton: CRC Press, 2016.

THRALL, M. A.. Hematologia e Bioquímica Clínica

Veterinária. São Paulo: Roca, 2007.

TIZARD, I. R.. Imunologia Veterinária. 9 ed. Rio de Janeiro: Elsevier, 2014.

TOIT, P. J.. A new piroplasm (Sauroplasma thomasi ng, n. sp.) of a lizard (Zonurus giganteus, Smith). Onderstepoort J Vet Sci Anim Ind, v.9, p.289-299, 1937.

UILENBERG, G.; BLANC, C. P.. Note sur un Hematozoaire d'un Reptile malgache, Uroplatus fimbriatus (Gekkonidae): Sauroplasma sp. (Protozoa incertaesedis). Ann Parasitol Hum, v.41, p.209-212, 1966.

VAN, A. S. J.. Ecology, taxonomy and possible life cycles of blood protozoans infecting crag lizards (pseudocordylus spp.) From the eastern free state highlands. Thesis (Ph.D. (Zoology and Entomology) - University of the Free State, 2012.

YORKE, J. A.; NATHANSON, N.; PIANIGIANI, G.; MARTIN, J. Seasonality and the requirements for perpetuation and eradication of viruses in populations. American Journal of Epidemiology, v.109, n.2, p.103-123, 1979.

A CBPC - Companhia Brasileira de Produção Científica (CNPJ: 11.221.422/0001-03) detém os direitos materiais desta publicação. Os direitos referem-se à publicação do trabalho em qualquer parte do mundo, incluindo os direitos às renovações, expansões e disseminações da contribuição, bem como outros direitos subsidiários. Todos os trabalhos publicados eletronicamente poderão posteriormente ser publicados em coletâneas impressas sob coordenação da Sustenere Publishing, da Companhia Brasileira de Produção Científica e seus parceiros autorizados. Os (as) autores (as) preservam os direitos autorais, mas não têm permissão para a publicação da contribuição em outro meio, impresso ou digital, em português ou em tradução. 